\title{
Os produtos naturais são uma alternativa para o tratamento da candidose oral? Uma revisão de ensaios clínicos
}

\author{
Are natural products an alternative for the treatment of oral candidosis? A review of clinical trials \\ ¿Los productos naturales son una alternativa para el tratamiento de la candidiasis oral? Una revisión de los ensayos clínicos \\ Diego Romário SILVA ${ }^{1}$ \\ Rafaela Durrer Parolina CARVALHO ${ }^{1}$ \\ Ana Caroline RODRIGUES ${ }^{2}$ \\ Kaiane Tavares PONTES ${ }^{3}$ \\ Andréa Cristina Barbosa da SILVA \\ ${ }^{1}$ UNICAMP - Universidade Estadual de Campinas, Faculdade de Odontologia de Piracicaba, 13414-903 Piracicaba-SP, Brasil \\ ${ }^{2}$ UFCG - Universidade Federal de Campina Grand, 58429-900 Campina Grande-PB, Brasil \\ ${ }^{3}$ UEPB - Universidade Estadual da Paraíba, Centro de Ciências Biológicas e da Saúde, 58429-500 Campina Grande-PB, Brasil
}

\section{Resumo}

Introdução: a candidose oral é uma doença oportunista que acomete principalmente pacientes imunossuprimidos e usuários de prótese dentária total. Apesar da eficácia terapêutica dos antifúngicos utilizados atualmente para o tratamento desta doença, essas drogas apresentam muitos efeitos adversos relacionados à dose. Além disso, tem-se observado um aumento da resistência microbiana para esses agentes. Baseado nisso, uma fonte de busca promissora de princípios ativos alternativos para o tratamento da candidose oral são os produtos naturais. Objetivo: revisar a literatura sobre ensaios clínicos com produtos naturais para o tratamento da candidose oral, a fim de responder se existe evidência que os produtos naturais podem ser utilizados como tratamento alternativo para esta doença. Material e método: foi realizada uma síntese de todos os ensaios clínicos com produtos naturais para tratamento da candidose indexados banco de dados Pubmed. Resultado: seguindo os critérios de inclusão e exclusão entraram nesta revisão 6 estudos. Os estudos avaliaram a eficácia dos produtos naturais quanto aos parâmetros clínicos e micológicos e grau de satisfação do paciente. Conclusão: apesar da pouca quantidade de ensaios clínicos nesta temática, há evidência de que os produtos naturais podem ser usdos para tratar a candidose oral, especialmente alho, mamona, româ e melaleuca.

Descritores: Produtos Biológicos; Candida; Imunidade.

\section{Abstract}

Introduction: oral candidiasis is an opportunistic disease that mainly affects immunosuppressed patients and users of total dental prosthesis. Despite the therapeutic efficacy of currently used antifungals for the treatment of this disease, these drugs have many dose related adverse effects. In addition, an increase in microbial resistance has been observed for these agents. Based on this, a promising source of alternative active principles for the treatment of oral candidosis are the natural products. Objective: to review the literature on clinical trials with natural products for the treatment of oral candidosis in order to answer if there is evidence that natural products can be used as an alternative treatment for this disease. Material and method: a synthesis of all clinical trials with natural products for the treatment of candidiasis indexed Pubmed database was performed. Results: following the inclusion and exclusion criteria, 6 studies were included in this review. The studies evaluated the efficacy of the natural products regarding clinical and mycological parameters and degree of patient satisfaction. Conclusion: despite the limited amount of clinical trials on this subject, there is evidence that natural products can be used to treat oral candidosis, especially garlic, castor oil, pomegranate, and melaleuca.

Descriptors: Biological Products; Candida; Immunity.

\section{Resumen}

Introducción: la candidose oral es una enfermedad oportunista que afecta principalmente a los pacientes inmunosuprimidos ya los usuarios de prótesis dentales totales. A pesar de la eficacia terapéutica de los antifúngicos utilizados actualmente para el tratamiento de esta enfermedad, estos medicamentos presentan muchos efectos adversos relacionados con la dosis. Además, se ha observado un aumento de la resistencia microbiana para estos agentes. En base a esto, una fuente de búsqueda prometedora de principios activos alternativos para el tratamiento de la candidiasis oral son los productos naturales. Objetivo: revisar la literatura sobre ensayos clínicos con productos naturales para el tratamiento de la candidiasis oral, a fin de responder si existe evidencia que los productos naturales pueden ser utilizados como tratamiento alternativo para esta enfermedad. Material y método: se realizó una síntesis de todos los ensayos clínicos con productos naturales para el tratamiento de la candidose indexada base de datos Pubmed. Resultado: siguiendo los criterios de inclusión y exclusión entraron en esta revisión 6 estudios. Los estudios evaluaron la eficacia de los productos naturales en cuanto a los parámetros clínicos y micológicos y grado de satisfacción del paciente. Conclusión: a pesar de la poca cantidad de ensayos clínicos en esta temática, hay evidencia de que los productos naturales pueden ser utilizados para tratar la candidose oral, especialmente ajo, ricino, granada y melaleuca.

Descriptores: Productos Biológicos; Candida. Inmunidad.

\section{INTRODUÇÃO}

A candidose é uma doença fúngica parasitária causada por espécies do gênero Candida. Trata-se de uma infecção de amplo espectro, podendo ocorrer de forma local (mucocutânea) ou sistêmica. O tipo mucocutâneo é uma das infecções oportunistas mais ocorrentes em pacientes imunocomprometidos e acometidos por câncer ${ }^{1}$. Essa condição se manifesta também, com frequência, e em diferentes graus, nos usuários de prótese dentária total ${ }^{2}$.

A candidose oral apresenta formas clínicas variadas: forma aguda, crônica e em associação com outros tipos de lesões ${ }^{3}$. Embora já tenha sido considerada uma doença de idosos ou bebês, aceitase atualmente sua ocorrência na população em geral ${ }^{4}$.
Dentre as espécies de Candida isoladas da cavidade oral, destaca-se a Candida albicans ${ }^{5}$. É a espécie mais presente no ambiente bucal, em humanos, ocorrendo na boca de até $80 \%$ dos indivíduos saudáveis ${ }^{6}$. Alterações no ambiente bucal podem favorecer o crescimento de Candida, causando um desequilíbrio que pode levar esse microrganismo de um estado comensal para um estado patogênico. Na maioria das vezes, essas alterações estão relacionadas a desequilíbrios imunológicos do hospedeiro ${ }^{7}$.

Com relação ao tratamento da candidose oral, é possível destacar fármacos, como, derivados azólicos (fluconazol, miconazol e itraconazol) e os 
poliênicos (nistatina e anfotericina $\mathrm{B})^{8}$. Apesar da boa ação terapêutica encontrada nos grupos químicos de antifúngicos conhecidos, tais drogas podem apresentar efeitos indesejáveis, onde é possível destacar a toxicidade relacionada com a dose. Essa é a principal limitação para o uso desses agentes e também a principal justificativa para a busca de novas formulações de antifúngicos com atividade terapêutica adequada. Desse modo, um agente antifúngico ideal deve ter toxicidade nula ou reduzida em relação às células humanas ${ }^{9}$. Uma fonte de busca para essas possíveis propriedades e desenvolvimento de novas drogas pode ser obtida por meio dos produtos naturais de origem vegetal. Dessa forma, o objetivo da presente pesquisa foi analisar, descritivamente, por meio de revisão sistemática, os estudos que utilizaram produtos naturais para o tratamento de candidose oral, com a finalidade fornecer informações acerca de terapias alternativas para esta doença oportunista.

\section{MATERIAL E MÉTODO}

\section{- Pergunta de estudo}

Esta revisão sistemática foi realizada para abordar a questão específica: Existe evidência científica para o uso de produtos naturais no tratamento da candidíase oral?

\section{- Estratégia de busca}

Foi realizada uma revisão sistematizada da literatura na base de dados The National Library of Medicine, Washington DC (MEDLINE - PubMed). Para conduzir a pesquisa foi utilizada ferramenta de busca «advanced», disponível no site NCBI, a partir das combinações dos descritores apresentados na Tabela 1.

Tabela 1. Estratégia de busca e bases de dados bibliográficas usadas para recuperar os artigos para esta revisão

\begin{tabular}{|c|c|}
\hline $\begin{array}{l}\text { te } \mathrm{de} \\
\text { rência }\end{array}$ & $\begin{array}{l}\text { Estratégia de busca, incluindo descritores } \\
\text { combinações com operadores booleanos }\end{array}$ \\
\hline $\begin{array}{l}\text { Medline } \\
\text { via } \\
\text { Pubmed } \\
\text { (Since } \\
1966 \text { ) }\end{array}$ & 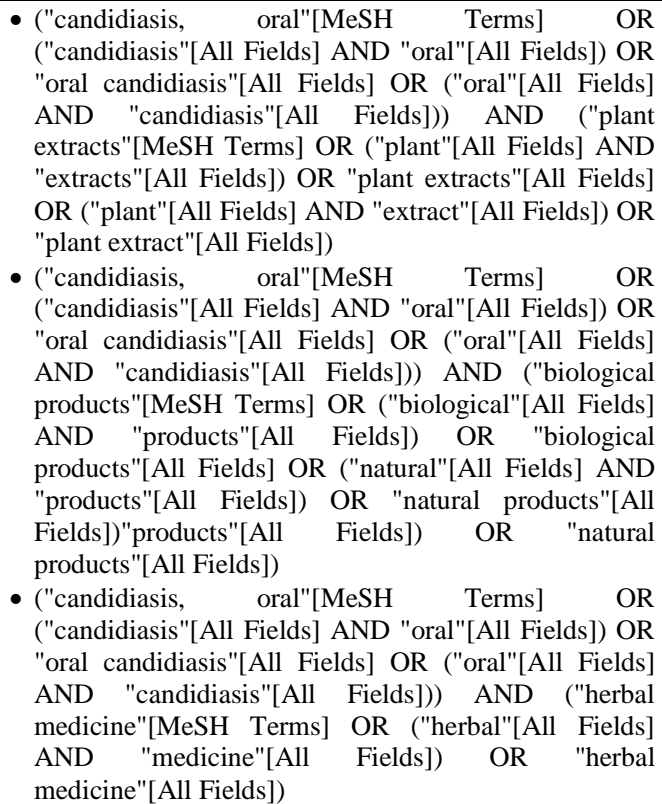 \\
\hline
\end{tabular}

\section{- Critérios de elegibilidade}

Uma triagem sistemática dos artigos foi realizada por dois examinadores dependentes de acordo com critérios de inclusão apresentados na Figura 1. A busca resultou em 235 artigos. Apenas ensaios clínicos foram selecionados. Após revisão dos títulos e resumos, foram encontrados 245 artigos, os quais foram avaliados quando aos seguintes critérios de inclusão: artigo do tipo ensaio clínico, que abordem o tratamento de candidose oral com produtos naturais, submetidos a um comitê de ética em pesquisa com seres humanos.

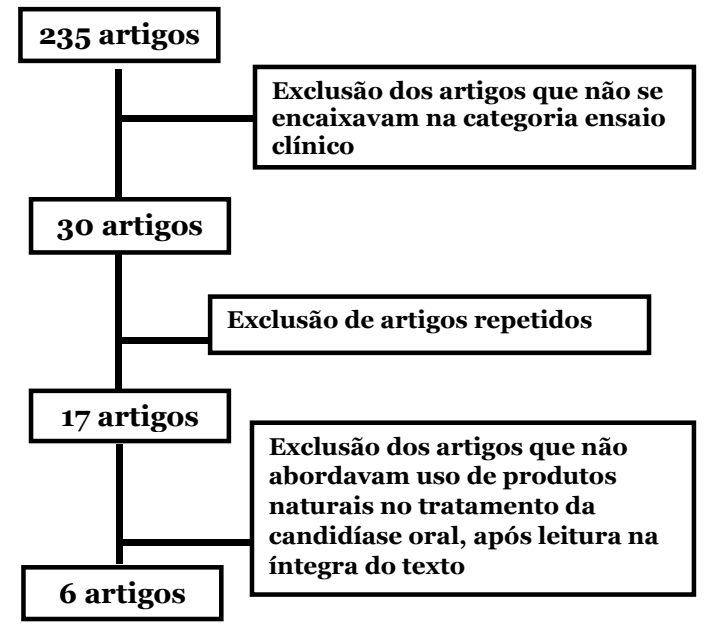

Figura 1: Esquematização da estratégia de seleção dos artigos de acordo com os critérios de elegibilidade.

\section{RESULTADOS E DISCUSSÃO}

De acordo com a estratégia previamente definida, foram encontrados 235 artigos, dos quais 6 atenderam aos critérios de inclusão e foram incluídos na revisão final após análise minuciosa $^{10-15}$ (Tabela 2).

A Tabela 2 traz os estudos em ordem cronológica de publicações, com informações relevantes sobre a metodologia e a contribuição do estudo com informações para assegurar o uso dos produtos naturais no tratamento da candidose oral. Os estudos incluídos nesta revisão avaliaram a eficácia de produtos naturais de duas formas, por meio a avaliação clínica das lesões (eritema) e/ou exame laboratorial para avaliação de do crescimento de $C$. albicans de amostras retiradas do local da lesão.

Com o objetivo de avaliar a eficácia da solução oral de Melaleuca no tratamento de candidose orofaríngea resistentes ao fluconazol, em pacientes com AIDS, Jandourek et al. ${ }^{10}$ realizaram ensaio clínico não randomizado. Um total de 13 pacientes foram incluídos no estudo. Apenas 12 pacientes foram avaliados. $\mathrm{Na}$ avaliação após o tempo de 2 semanas, sete dos 12 pacientes melhoraram, nenhum foi curado, e seis permaneceram inalterados. Na avaliação no tempo de 4 semanas, oito dos 12 pacientes mostraram uma 
resposta (dois foram curados e seis apresentaram melhora), quatro não responderam ao tratamento e um teve agravamento. Uma avaliação de seguimento 2 a 4 semanas após a interrupção da terapia revelou que não houve recidivas clínicas nos dois pacientes que foram curados. Apesar da eficácia clínica em dois pacientes, o estudo não foi randomizado, não apresentou análise estatística bem como não mencionam a recomendação da solução testada para o tratamento da candidose oral.

Tabela 2. Ensaios clínicos que utilizaram produtos naturais para o tratamento da candidose oral, incluídos nesta revisão

\begin{tabular}{|c|c|c|c|c|c|c|c|c|}
\hline Produto natural & $\begin{array}{l}\text { Estudo } \\
\text { randomizado }\end{array}$ & Placebo & $\begin{array}{l}\text { Método } \\
\text { avaliação }\end{array}$ & de & $\begin{array}{l}\text { Eficácia } \\
\text { estatisticamente } \\
\text { significativa }\end{array}$ & $\begin{array}{l}\text { Efeito } \\
\text { adverso }\end{array}$ & $\begin{array}{l}\text { Recomendação pelos } \\
\text { autores para utilização } \\
\text { como } \\
\text { alternativa }\end{array}$ & Autor/Ano \\
\hline $\begin{array}{l}\text { Melaleuca } \\
\text { (Melaleuca } \\
\text { alternifólia) }\end{array}$ & Não & Não & $\begin{array}{l}\text { Exame clínico } \\
\text { laboratorial } \\
\text { micológico }\end{array}$ & $\mathrm{e}$ & - & Sim & - & $\begin{array}{l}\text { Jandourek et al. }{ }^{10} \\
1998\end{array}$ \\
\hline $\begin{array}{l}\text { Româ } \\
\text { granatum) }\end{array}$ & Sim & Não & $\begin{array}{l}\text { Exame clínico } \\
\text { laboratorial } \\
\text { micológico }\end{array}$ & $\mathrm{e}$ & Sim & Não & - & $\begin{array}{l}\text { Vasconcelos et al. }{ }^{11} \\
2003\end{array}$ \\
\hline $\begin{array}{l}\text { Melaleuca } \\
\text { (Melaleuca } \\
\text { alternifólia) }\end{array}$ & Sim & Sim & Exame clínico & & Sim & Não & - & $\begin{array}{l}\text { Catalán et al.12 } \\
2008\end{array}$ \\
\hline $\begin{array}{l}\text { Limão (Citrus } \times \\
\text { limon) e Capim- } \\
\text { limão } \\
\text { (Cymbopogon } \\
\text { citratus) }\end{array}$ & Sim & Não & Exame clínico & & Não & Sim & - & $\begin{array}{l}\text { Wright et al. }{ }^{13} \\
2009\end{array}$ \\
\hline $\begin{array}{l}\text { Alho (Allium } \\
\text { sativum) }\end{array}$ & Sim & Não & $\begin{array}{l}\text { Exame clínico e gra } \\
\text { de satisfação } \\
\text { paciente. }\end{array}$ & & Sim & Não & Sim & $\begin{array}{l}\text { Bakhshi et al. }{ }^{14} \\
2012\end{array}$ \\
\hline $\begin{array}{l}\text { Mamona (Ricinus } \\
\text { communis) }\end{array}$ & Sim & Não & $\begin{array}{l}\text { Exame clínico } \\
\text { laboratorial } \\
\text { micrológico }\end{array}$ & $\mathrm{e}$ & Não & Não & Sim & $\begin{array}{l}\text { Pinelli et al. }{ }^{15} \\
2013\end{array}$ \\
\hline
\end{tabular}

Outro ensaio clínico foi realizado com uma formulação de melaleuca com condicionadores teciduais (Fitt, Lynal, Coe-Comfort), por Catalan et al. ${ }^{12}$. Foram avaliados três grupos de 9 pacientes com estomatite protética quanto à resposta ao tratamento de melaleuca+ Coe-Comfort, nistatina+ Coe-Comfort e apenas Coe-Comfort. Os pacientes tratados com M. alternifolia misturado com Coe-Comfort mostraram uma diminuição significativa na inflamação palatal em comparação com aqueles tratados com Coe Comfort $(\mathrm{P}=0,001)$. Os autores concluem que o óleo de melaleuca é eficaz para o tratamento da candidose oral, embora não opinem quanto a sua indicação como tratamento alternativo.

Vasconcelos et al. ${ }^{11}$ avaliaram a eficácia de um gel incorporado com româ em 30 pacientes com estomatite protética, durante 15 dias. Um grupo controle, de 30 pacientes, tratado com miconazol foi adicionado ao estudo para parâmetro de comparação. Ambos os tratamentos foram avaliados quanto a melhora clínica e a positividade para Candida. Os resultados mostraram uma resposta clínica satisfatória e em 21 indvíduos tratados com o gel de româ e (23 indivíduos com resultado negativo para Candida) e 27 indivíduos tratados com miconazol (25 indivíduos com resultado negativo para Candida). Os autores indicam que o gel de româ pode ser uma alternativa terapêutica associada ao tratamento da estomatite protética. É bem postulado na literatura que a româ apresenta atividade antimicrobiana contra muitos microrganismos orais, entre eles espécies de Candida ${ }^{16}$. Dessa forma, a falta de um grupo tratado apenas com o extrato de româ dificulta o entendimento da importância da formulação em gel.

Wright et al. ${ }^{13}$ investigaram a eficácia e segurança da utilização de limão e capim-limão no tratamento de candidose oral em pacientes HIV positivos. Um total de 90 pacientes fora randomizados para serem tratados com limão, capimlimão e violeta genciana. Nenhum dos grupos foi considerado clinicamente tratado ao final do experimento. Porém, o suco de limão apresentou melhores resultados que a solução aquosa de genciana $0,5 \%$ no tratamento $(\mathrm{p}<0,02)$. Os grupos tratados com capim-limão e violeta genciana também mostraram alguma melhora clínica $(\mathrm{p}<0,05)$. Os autores consideram validado o suco de limão e capim-limão para o tratamento de candidose em HIV positivos, embora os resultados indiquem que o suco de limão e capim-limão poderiam ser utilizados como um adjuvante a uma terapia já estabelecida para o tratamento da candidose oral, uma vez que os pacientes não tenham de fato sido tratados ao final do experimento.

Para efeito de comparação entre a eficácia da nistatina e do alho no tratamento da candidose oral, Bakhshi et al. ${ }^{14}$ avaliaram 40 pacientes divididos para os dois tipos de tratamento, por um período de quatro semanas. Os resultados mostraram que ambos os tratamentos resultaram em recuperação significativa ( $p<0,0001$ ), porém uma recuperação acelerada foi demonstrada para a nistatina $(\mathrm{p}<0,001)$. Uma maior satisfação com o uso de alho, em vez de nistatina foi observado ( $\mathrm{p}<0,0001)$. Os autores concluíram que devido a eficácia do alho e sua ausência de efeitos adversos, ele pode ser indicado como terapia alternativa para candidose oral. Este foi o único estudo incluso nesta revisão que avaliou, além da eficácia clínica do produto natural, o grau de satisfação do paciente. Esta variável é extremamente importante, uma vez que alguns tratamentos bastante eficazes trazem efeitos indesejáveis ao paciente. 
Já Pinelli et al. ${ }^{15}$ avaliaram a eficácia da mamona em comparação com a nistatina e o miconazol no tratamento da estomatite protética. Avaliações clínicas e micológicas foram realizadas antes, 15 e 30 dias após o tratamento. Embora nenhum dos tratamentos tenha diminuido o número de UFC/mL (Unidades Formadoras de Colônia por $\mathrm{mL}$ - avaliação micológica), os autores concluiuram que o a mamona pode melhorar a condição clínica de estomatite protética, com resultados semelhantes ao miconazol.

Apesar de haver muitos estudos in vitro que avaliem a atividade antimicrobiana de produtos naturais contra espécies de Candida $^{17,18}$, os ensaios clínicos ou até mesmo testes in vivo com animais que mostrem a eficácia terapêutica do produto e a sua segurança ainda são incipientes. Dado o pouco arsenal antifúngico atualmente existente ${ }^{19}$ e o aumento na resistência dos das espécies de Candida ${ }^{20,21}$, a pouca quantidade de estudos clínicos que evidenciem novas alternativas terapêuticas é preocupante. É preciso investir mais em estudos in vivo e principalmente em ensaios clínicos.

Com base nos estudos incluídos nesta revisão, é perceptível a falta de padronização quanto a realização de ensaios clínicos com produtos naurais para o tratamento da candidose oral. A maioria dos estudos não indica diretamente a utilização do produto natural investigado em seu estudo para sua utilização como terapia alternativa para o tratamento dessa doença oportunista. Os estudos que avaliaram parâmetros clínicos e micológicos ${ }^{10,11,15}$ parecem ter maior segurança na apresentação dos seus resultados. Porém um estudo que se destacou foi o de Bakhshi et al. ${ }^{14}$ que avaliaram a variável grau de satisfação do paciente com o tratamento. Baseado nisso, um ensaio clínico ideal para responder a questão da eficácia dos produtos naturais como terapia alternativa para o tratamento da candidose oral deveria incluir a variável clínica, micológica e grau de satisfação do paciente. Nenhum dos estudos incluídos nesta revisão apresentou estes três parâmetros juntos. Apesar disso, há evidência de que a utilização de alguns produtos naturais pode ser bem sucedida como terapia alternativa no tratamento da candidose oral, especialmente a variável estomatite protética.

\section{CONCLUSÃO}

Apesar dos poucos ensaios clínicos com produtos naturais para o tratamento da candidose oral, alguns produtos naturais apresentaram resultados semelhantes a antifúngicos e com a vantagem de apresentar pouco ou nenhum efeito adverso. Dessa forma há evidência de que os produtos naturais, especialmente alho, mamona, româ e melaleuca na sua forma de extrato bruto ou em formulações podem ser utilizados para o tratamento da candidose oral. Porém, estudos complementares são necessários para definir com precisão posologia, mecanismo de ação e testes de sensibilidade para diferentes espécies do gênero Candida.

\section{REFERÊNCIAS}

1. Hani U, Shivakumar HG, Vaghela R, Osmani RAM, Shrivastava A. Candidiasis: A fungal infection- current challenges and progress in prevention and treatment. Infect Disorders Drug Targets. 2015;15(1):42-52.

2. Pereira CA, Domingues N, Araújo MI, Junqueira JC, Back-Brito GN, Jorge AO. Production of virulence factors in Candida strains isolated from patients with denture stomatitis and control individuals. Diagn Microbiol Infect Dis. 2016;85(1):66-72.

3. Patil S, Rao RS, Majumdar B, Anil S. Clinical appearance of oral candida infection and therapeutic strategies. Front Microbiol. 2015;6:1391.

4. Dos Santos AL. HIV aspartyl protease inhibitors as promising compounds against Candida albicans. World J Biol Chem.2010;1(2):21-30.

5. Shao LC, Sheng CQ, Zhang WN. Recent advances in the study of antifungal lead compounds with new chemical scaffolds. Yao Xue Xue Bao. 2007;42(11):1129-36.

6. Kullberg BJ, Arendrup MC. Invasive candidiasis. N Engl J Med. 2015. 373:1445-56.

7. Williams D, Lewis M. Pathogenesis and treatment of oral candidosis. J Oral Microbiol. 2011;3:5771. doi: 10.3402/jom.v3i0.5771.

8. Kuriyama T, Williams DW, Bagg J, Couçter WA, Ready D, Lewis MAO. In vitro susceptibility of oral Candida to seven antifungal agents. Oral Microbiol Immunol. 2005;20(6):349-53.

9. Wong SS, Samaranayake LP, Andseneviratne CJ. In pursuit of the ideal antifungal agent for Candida infections: high-throughput screening of small molecules. Drug Discov Today. 2014;19(11):1721-30.

10.Jandourek A, Vaishampayan JK, Vazquez JA. Efficacy of melaleuca oral solution for the treatment of fluconazole refractory oral candidiasis in AIDS patients. AIDS. 1998;12(9):1033-37.

11.Vasconcelos LC, Sampaio MC, Sampaio FC, Higino JS. Use of Punica granatum as an antifungal agent against candidosis associated with denture stomatitis. Mycoses. 2003;46(56):192-96.

12.Catalán A, Pacheco JG, Martínez A, Mondaca MA. In vitro and in vivo activity of Melaleuca alternifolia mixed with tissue conditioner on Candida albicans. Oral Surg Oral Med Oral Pathol Oral Radiol Endod. 2008;105(3):327-32.

13. Wright SC, Maree JE, Sibanyoni M. Treatment of 
oral thrush in HIV/AIDS patients with lemon juice and lemon grass (Cymbopogon citratus) and gentian violet. Phytomedicine. 2009; 16(2/3):118-24.

14.Bakhshi M, Taheri JB, Shabestari SB, Tanik A, Pahlevan R. Comparison of therapeutic effect of aqueous extract of garlic and nystatin mouthwash in denture stomatitis. Gerodontology. 2012;29(2):e680-84.

15.Pinelli LA, Montandon AA, Corbi SC, Moraes TA, Fais LM. Ricinus communis treatment of denture stomatitis in institutionalised elderly. J Oral Rehabil. 2013;40(5):375-80.

16.Pai MB, Prashant GM, Murlikrishna KS, Shivakumar KM, Chandu GN. Antifungal efficacy of Punica granatum, Acacia nilotica, Cuminum cyminum and Foeniculum vulgare on Candida albicans: an in vitro study. Indian J Dent Res. 2010;21(3):334-36.

17.Höfling JF, Anibal PC, Obando-Pereda GA, Peixoto IA, Furletti VF, Foglio MA et al. Antimicrobial potential of some plant extracts against Candida species. Braz J Biol. 2010;70(4):1065-68.

18.Doddanna SJ, Patel S, Sundarrao MA, Veerabhadrappa RS. Antimicrobial activity of plant extracts on Candida albicans: an in vitro study. Indian J Dent Res. 2013;24(4):401-5.

19. Maubon D, Garnaud C, Calandra T, Sanglard D, Cornet M. Resistance of Candida spp. to antifungal drugs in the ICU: where are we now? Intensive Care Med. 2014; 40(9):1241-55.

20. Alexander BD, Johnson MD, Pfeiffer CD, Jiménez-Ortigosa C, Catania J, Booker R et al. Increasing echinocandin resistance in Candida glabrata: clinical failure correlates with presence of FKS mutations and elevated minimum inhibitory concentrations. Clin Infect Dis. 2013;56(12):1724-32.

21. Shields RK, Nguyen MH, Press EG, Updike CL, Clancy CJ. Caspofungin MICs correlate with treatment outcomes among patients with Candida glabrata invasive candidiasis and prior echinocandin exposure. Antimicrob Agents Chemother. 2013;57(8):3528-35.

\section{CONFLITO DE INTERESSES}

Os autores declaram não haver conflitos de interesse.

\section{AUTOR PARA CORRESPONDENCIA}

\section{Diego Romário Silva}

diegoromarioo@gmail.com.br

Submetido em 25/05/2018

Aceito em 27/06/2018 